\title{
Aortic Anastomosis of Graft
}

National Cancer Institute

\section{Source}

National Cancer Institute. Aortic Anastomosis of Graft. NCI Thesaurus. Code C99915.

The connection where the graft joins the aorta usually less than or equal to $3 \mathrm{~mm}$ from insertion point. (ACC) 